\title{
Novel Modeling of the Phenomenon of Air-Cooled Solid
}

\author{
Zdzisław Pluta, Tadeusz Hryniewicz* \\ Koszalin University of Technology, Raclawicka 15-17, PL 75-620 Koszalin, Poland \\ *E-mail address: Tadeusz.Hryniewicz@tu.koszalin.pl
}

\begin{abstract}
The paper turns the attention to the thermal problems of the solid cooling in air. The physical aspect of mathematic approach has been underlined. The analysis and critical evaluation of Newton's Law concerning the solid air-cooling have been performed. The cognitive path leading to the solution is presented with the result being the body temperature dependence on time of cooling down in the air. Then the course of solution concerning the result of the adequate dependence of temperature of the cooled body on time has been presented. In the study, the stress is put on constant magnitudes which characterize the susceptibility of solid to the cooling in the air. In the end, the synthetic formulation of all functional characteristics of the thermal phenomenon discussed in the paper, have been presented.
\end{abstract}

Keywords: Phenomenon of body cooling; Thermal space-time; Potential field; Time constant; Cooling rate; Temperature; Time

\section{INTRODUCTION}

In science, there is a need to return to the sources of natural reality. It appears in many cases the descriptions of the nature are inadequate in their character. That means they do not correspond with the studied reality. In fact, they do not regard the initial conditions, corresponding with the beginning of the phenomenon.

Inadequate character of the description of natural phenomena results also from a common tendency to linearize their courses. That means, the non-linear characteristics are substituted by a straight line, with the result called zero- or first approach, as noticed e.g. by [1].

Following such simplifications, lots of ways of linearizations, not justifiable from the scientific point of view, have been elaborated. Many of them have been presented, for instance in [2], and they are called as: common, harmonic, energetic, simple, balanced, statistic, acc. to the criterion of minimum of mean-square deviation, or acc. to the distribution function.

The author of the work [3] characterizes these simplifying actions with a very strong remark: „It is a heresy to justify the attempts to use the linear superposition for non-linear systems". The philosophy of approach to the studies of non-linear phenomena is discussed there with a suggestion to consider the „black box" method; lack of non-linear logistics is also stressed in the paper [3].

Another approach concerns the linearization on the ground of Newton's mechanics. Here the linear course of velocity is assumed by calling this as the uniformly variable motion, with the initial velocity being zero. Thus the variable motion, or by the model as uniformly 
variable, is described beginning from the second kinetic link. Then the actions were directed into two reverse directions. To obtain the dependence of path length on time, the velocity function was integrated. The differentiation, as the action reversal to integrating, resulted in getting the record on acceleration. Unfortunately, that acceleration appears to be constant, invariable; getting further magnitudes is impossible, because all of them as the consecutive derivatives, equal simply zero.

The next reason for the inadequacy of the created descriptions of phenomena is physically unreasonable acceptance the forms of the regression functions, assuming the characteristics of real systems. Thus obtained the empirical (statistic-experimental) formulae include some constants (coefficients, power exponents), having no physical sense, proving of the degree of fitting of a theoretical curve only to the conglomeration of experimental points.

Some illustration of such actions, on the ground of science, may be the attempts to describe the configuration of material cutting traces by means of a tool of a determined elastic beam: abrasive, water-abrasive, cryogenic, laser, electron, or plasma. It appears the descriptions of geometry of these traces are quite differentiated and still do not reflect a real outline/profile. There were some attempts to describe the cut-off by a parabola $[4,5]$; logarithmic spiral $[6,7]$, as well as polynomial of the third degree $[8,9]$.

They are just some examples sufficiently well illustrating the considered problem of returning to the source. Some more examples of these quests will be delivered concerning the title work problem.

\section{NEWTON'S LAW ON THE COOLING RATE OF A BODY IN AIR}

The well known Newton's law, treating the rate of the body cooling down in air, will be considered. The rate of this phenomenon is, according to the mentioned law, directly proportional to the temperature difference of: a solid and air. One should mention, that law is the example of using a differential equation of divided variables. It is then a fine illustration in the area of applications of solving this type of equation. That way it was used, for instance in the literature [10].

Here is the mentioned Newton's law:

$$
-\frac{d T}{d t}=k\left(T-T_{0}\right)
$$

where: $T$ - body temperature, $T_{0}$ - air temperature, $t$ - time, $\frac{d T}{d t}-$ the rate of body cooling in the air, $k$-constant coefficient, dependent on the type of cooled body.

For the illustration purposes, the equation may be written in the following form:

$$
v=k\left(T-T_{0}\right)=-k T_{0}+k T=v_{0}^{*}+k T
$$

with the symbol $v$ denoting just the rate of body cooling, and $v_{0}^{*}$ - initial, apparent rate of the phenomenon.

It is worth characterizing closer the coefficient $k$. For this purpose one may formulate the dependence $k=f(T)$. It has the following form: 


$$
k=\frac{v-v_{0}^{*}}{T}=\frac{v}{T-T_{0}}
$$

If the temperature is given in Celsius degree $\left[{ }^{\circ} \mathrm{C}\right]$, and the rate of body cooling expressed in the Celsius degree per second $\left[{ }^{\circ} C \cdot s^{-1}\right]$, so the unit of the coefficient $k$ will be $\left\lfloor s^{-1}\right\rfloor$, that results from the operations on the mentioned component units of magnitudes, that is

$$
[k]=\frac{{ }^{o} C \cdot s^{-1}}{{ }^{o} C}=s^{-1}
$$

That means, this coefficient is the gradient of the growth of cooling rate, thus possessing a determined physical interpretation.

The plot of dependence (2) is the straight line (Fig. 1), with the angular coefficient $k$, resulting from the equation (3), having a positive value. Only for the coordinates $T=T_{0}$, $v=0$ that coefficient is non-determined, with the non-determination of type 0:0. The physical character of that dependence corresponds with the continuous line for $T>T_{0}$; whereas the dashed line, in the interval $T \in\left\langle 0, T_{0}\right)$, illustrates mathematical, mathematically determined illustration of the course.

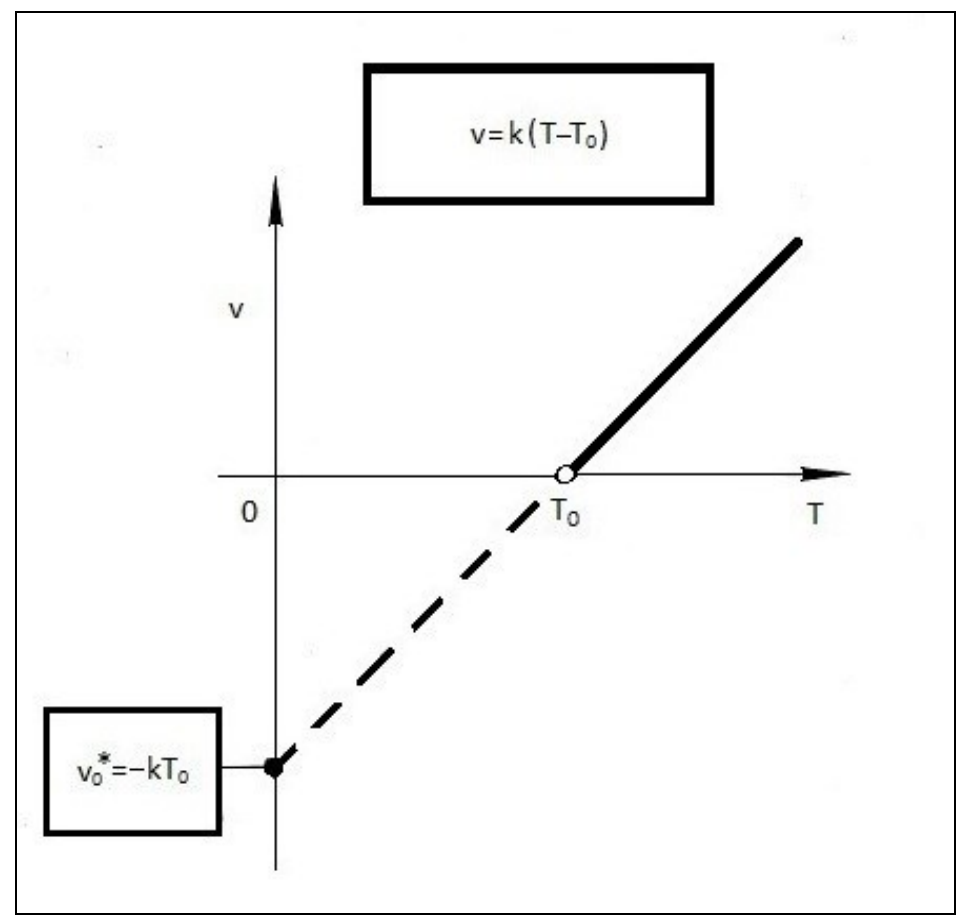

Fig. 1. Indicatrisse of the dependence of body cooling rate on its temperature, acc. to the Newton's Law.

The solution of equation (1) provides the formulation of response to the question on what is the lapse time $t^{*}$ the body will be cooled down to the temperature $T^{*}$, if its initial temperature equals $T_{1}$. Although the procedure leading to answer that question is generally known, it is worth presenting that here as the point of outcome to further considerations, directed into the source itself. 
The equation (1) may be written in the following form:

$$
\frac{d T}{T-T_{0}}=-k d t
$$

which, after the integrating on both sides, assumes the following configuration:

$$
\ln \left(T-T_{0}\right)=-k t+C^{*}
$$

or

$$
T-T_{0}=e^{-k t+C^{*}}=e^{C^{*}} \cdot e^{-k t}=C e^{-k t}
$$

From the condition, that in the moment $t=0$, the temperature $T=T_{1}$, one obtains

$$
C=T_{1}-T_{0}
$$

therefore, after substituting (7) to (6), one obtains the next form of the equation, namely

$$
T-T_{0}=\left(T_{1}-T_{0}\right) e^{-k t}
$$

and then

$$
t=\frac{1}{k} \ln \frac{T_{1}-T_{0}}{T-T_{0}}
$$

After substituting $T=T^{*}$ to the former formula, one obtains the searched time of cooling $t^{*}$, that is

$$
t^{*}=\frac{1}{k} \ln \frac{T_{1}-T_{0}}{T^{*}-T_{0}}
$$

That course of temperature of the body cooled in the air (8), being the solution of the Newton's law (1), has the character of exponentially decreasing curve (Fig. 2), coming out of the point corresponding with the initial temperature $T_{1}$ of the body. 


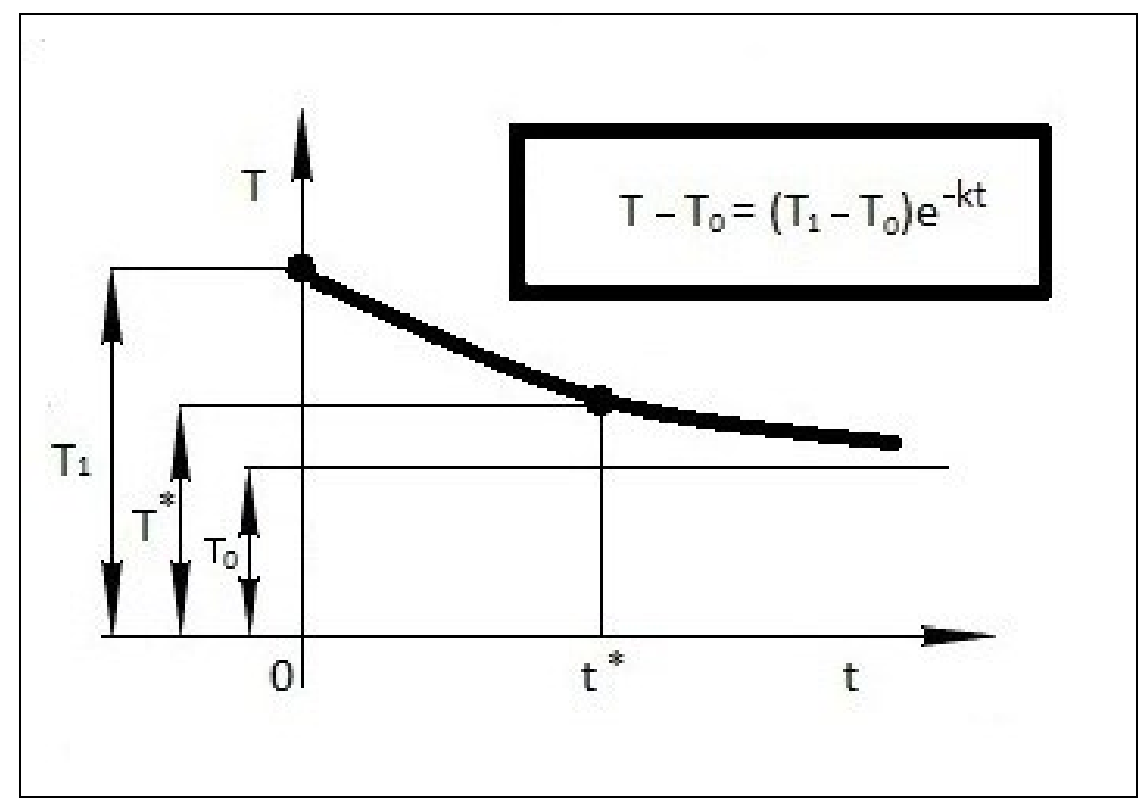

Fig. 2. Course of the body temperature cooled in air, corresponding with the Newton's Law.

The rate $v$ of the body cooling, being the derivative (with the minus sign) of function (8) against time $t$, that is $-\frac{d T}{d t}$, has the following form:

$$
v=-\frac{d T}{d t}=\frac{T_{1}-T_{0}}{k} e^{-k t}=v_{1} e^{-k t}
$$

and its graphical image (Fig. 3) is the exponentially decreasing curve, approaching the horizontal asymptote, placed on the time axis.

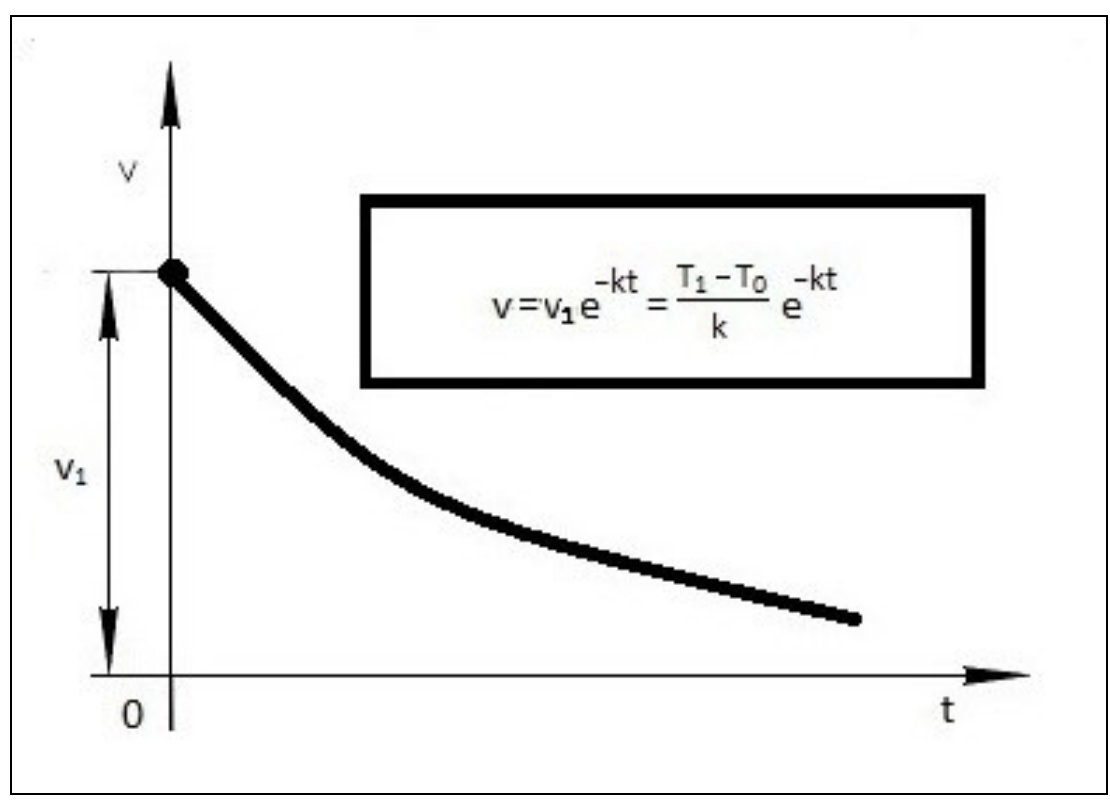

Fig. 3. Course of the dependence of the body cooling rate $v$ on temperature $t$ of the air, acc. to the Newton's Law. 
It is time now for a critical analysis of the outcome equation, which is the Newton's law, described by the differential equation (1). That equation is not adequate in character, that affects also its solution (8), as the consequence of imperfect content of the outcome equation. The graphical illustration (see, Fig. 2) clearly reveals that shortcoming. One may notice, the temperature of cooled body approaches the air temperature, being the horizontal asymptote, that is for the time $t \rightarrow \infty$ occurs $T \rightarrow T_{0}$. That means, if the time $t$ increases unlimitedly, the temperature $T$ tends to a complete value, corresponding with the air temperature $T_{0}$. Thus the temperatures never will be equaled, and that is inconsistent with the physical nature of the considered phenomena.

Hence, a return to the source is needed, which is the general outcome differential equation, describing the considered phenomenon of the body cooling. To say even more, that outcome form of the equation has a much broader application concerning any quantum changes of the natural reality. It is worth adding that the mentioned source form of the equation has been presented in [11]. There the detailed solutions of other examples of the technological systems are also delivered. The application of this source differential equation to the analytical description of the tool life under cut against the main velocity was recently presented in the work [12], and the kinetics of tool edge fixed flexibly was solved [13].

One may judge, the equation (1) has been formulated on the experimental basis only, may be with frequent numerous experimental points. It does not result from the analysis of the thermal reality/system, with the form proving of its irrelevancy.

\section{THERMAL EFFECTS UNDER THE SOURCE RECOGNITION}

At the source of cognitive way of the thermal reality there is a general differential equation, having the following form:

$$
d T= \pm \frac{\partial T}{\partial t} d t
$$

where the symbol $d T$ denotes the total differential of temperature of the cooled body, $d t-$ total differential of cooling time, $\frac{\partial T}{\partial t}$ - partial derivative of temperature against time. The signs $( \pm)$ are the algebraic operators, with $(+)$ possessing a formal significance only, because it confirms the physical sense of the determined record, whereas $(-)$ ascribes such a physical sense to the determined record. The description of the body cooling phenomenon requires using the operator $(-)$, for the temperature course as the function of time to be positive, with a physical sense.

Scheme of creation of adequate description of the dependence $T=f(t)$ is presented in Fig. 4 showing all elements of the reasoning process. The curve illustrating that dependence comes out of an initial point of the coordinates $t=0, T=T_{1}$, and further its course is decreasing exponentially. It completes its non-linear and real/physical course in the point 0 , where the body cooling phenomenon has its end; then the continuous real course is invariable, and constant. There is also further, non-real, fictional, just a mathematical fragment of the non-linear course (dashed line). The need for introducing such a further fragment of the curve will be explained soon. 


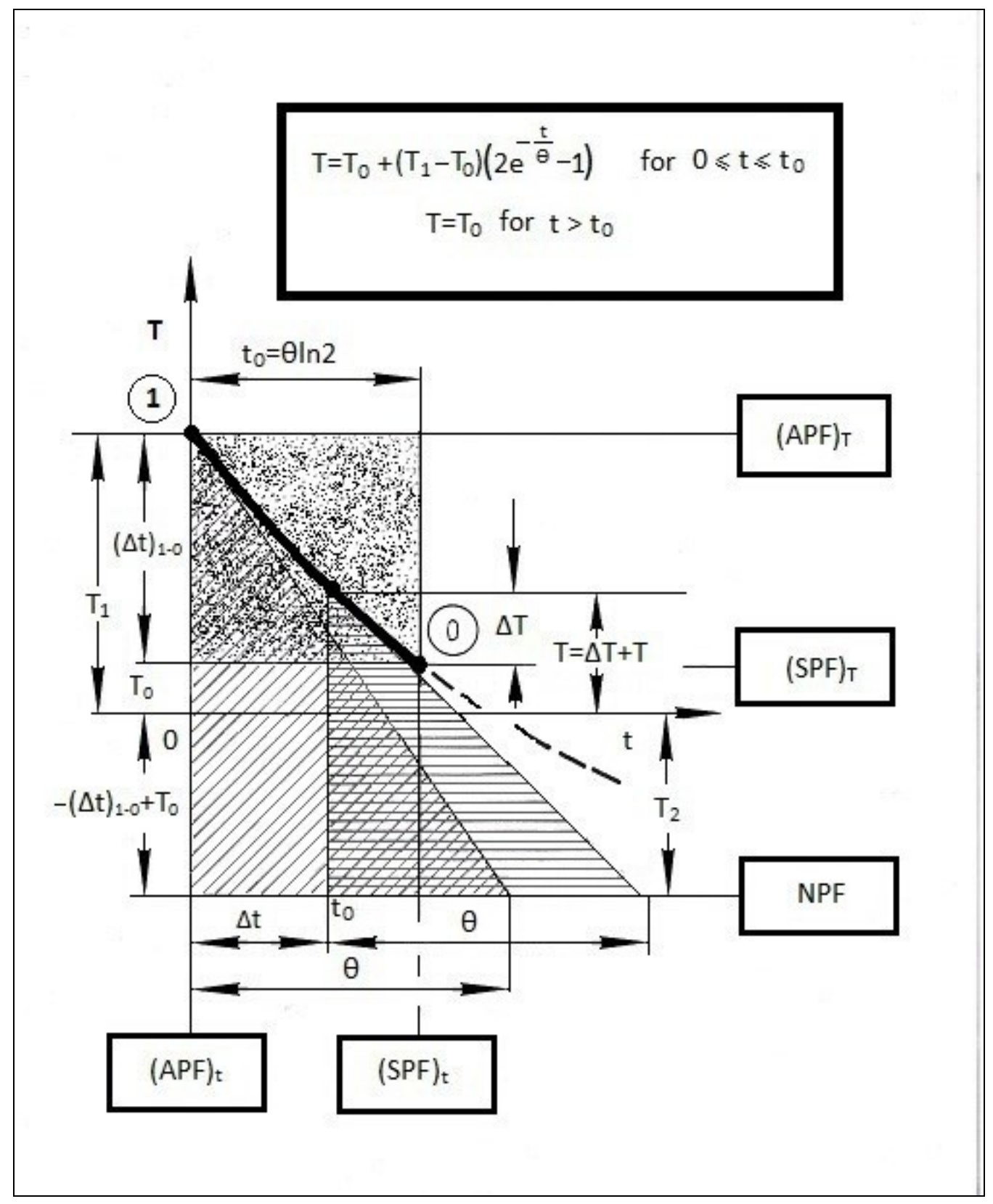

Fig. 4. Indicatrisse of adequate course of the dependence of temperature $T$ of the cooled body on time $t$ of its cooling.

Now the dependence (12) is to be integrated on both sides; one should remember that the total differential is the state function. The states, being the limits of integration, should be now determined.

The mentioned limits of the studies are the potential fields. These fields are situated on two directions; one of them is the direction of changes of temperature $T$, and the second one is the direction of time $t$. On the first direction the following fields may be differentiated: temperature unstable potential field $(A P F)_{T}$, temperature stable potential field $(S P F)_{T}$ and the nominal potential field $N P F$. On the second direction there are the following fields: time unstable potential field $(A P F)_{t}$, time stable potential field $(S P F)_{t}$. The fields $(A P F)_{T}$, $(S P F)_{T}$, and $(A P F)_{t},(S P F)_{t}$ confine the real thermal, physical space-time, as the proper one (dotted area); there the real, exponentially decreasing changes of temperature occur. 
Between the fields $(S P F)_{T}$ and $N P F$ there is an improper thermal space-time, fulfilling here the auxiliary role (it is like an auxiliary mathematical tool).

The temperature curve, comprised between the points $1-0$, is the envelope of rightangled triangles, moving with its horizontal leg on the nominal potential field; here this horizontal leg is invariable and equals the time constant $\Theta$, whereas the vertical leg is changing respectively, decreasing in due measure the displacement of the triangle in the time direction. That nominal field is situated symmetrically against the level of the end of cooling phenomenon, on the distance equal to the length of space-time in the temperature direction.

Therefore under the real/proper space-time that mentioned above, the improper spacetime is situated; there the curve (dashed line) approaches the asymptote, being that nominal potential field. This creation surely forms an auxiliary design, needed to describe the real curve, reflecting the adequate dependence of temperature of the cooled body on time.

It is worth adding that the considered phenomenon has a quantum nature. That means the thermal states vary in non-continuous way, by jump, or quantum, and between the states (marked by determined temperatures, that is the outcome temperature $T_{1}$ of the cooled body, and further, the final temperature $T_{0}$ of the body, equal the air temperature) a continuous heat exchange takes place. Those states take place on the described above the potential fields.

Now one may come to the integration of the equation (12). By integrating that equation, one should mark the limits of integrals from the total differentials. That means

$$
\int_{T_{2}}^{T} d T=-\frac{\partial T}{\partial t} \int_{\Delta t}^{\Delta t+\Theta} d t
$$

and further

$$
T-T_{2}=-\frac{d T}{d t} \Theta
$$

One may notice the partial derivative has been substituted by the quotient of total derivatives. It could be done that way because the total differentials have been clearly determined by introducing the limits of the integrals.

For comparison, it is worth presenting the equation (14) in the form corresponding with the form of equation (1), i.e. Newton's law. Here is the mentioned equation:

$$
-\frac{d T}{d t}=\frac{1}{\Theta}\left(T-T_{2}\right)
$$

It may be noted, it is quite similar to the equation (1) but substantially differing in content. Further, more distinct differences will be revealed during solving the equation (14), and its solution will indicate the anomalies contents.

Now let us determine the limits of the left integral of the equation (13) by introducing there the magnitudes $T_{0}, T_{1}$, and $\Delta T$. Thus 


$$
\int_{-(\Delta T)_{1-0}+T_{0}}^{T_{0}+\Delta T} d T=-\frac{\partial T}{\partial t} \int_{\Delta t}^{\Delta t+\Theta} d t
$$

and further

$$
(\Delta T)_{1-0}+\Delta T=\left(T_{1}-T_{0}\right)+\Delta T=-\frac{d T}{d t} \Theta
$$

or

$$
\frac{d T}{\left(T_{1}-T_{0}\right)+\Delta T}=-\frac{1}{\Theta} d t
$$

Furthermore, by integrating the equation (18) on both sides, one obtains the result as follows:

$$
\ln \left[\left(T_{1}-T_{0}\right)+\Delta T\right]=-\frac{1}{\Theta} t+C^{*}
$$

or

$$
\left(T_{1}-T_{0}\right)+\Delta T=e^{-\frac{t}{\Theta}+C^{*}}=e^{C^{*}} \cdot e^{-\frac{t}{\Theta}}=C e^{-\frac{t}{\Theta}}
$$

After considering that for $t=0$, the magnitude $\Delta T=T_{1}-T_{0}$, one obtains

$$
C=2\left(T_{1}-T_{0}\right)
$$

and after substituting (21) to (20), and then after considering that $\Delta T=T-T_{0}$

$$
T=T_{0}+\left(T_{1}-T_{0}\right)\left(2 e^{-\frac{t}{\Theta}}-1\right)
$$

The plot of the function (22) is the decreasing exponential curve (see Fig. 4). The segment of this plot (solid line), reflecting the illustration of the real phenomenon, runs through the real/physical thermal space-time. It terminates its variable course on the stable temperature potential field $(S P F)_{T}$, where further it keeps its energetic state. That means that for $t \geq t_{0}$ the temperature $T=T_{0}$ of the cooled body appears to be equal to the air temperature. 


\section{ANALYSIS OF THE DETAILED ADEQUATE DEPENDENCE OF THE COOLED BODY TEMPERATURE ON TIME OF COOLING}

In the frameworks of the analysis of equation (22) one should first determine the time $t_{0}$, corresponding with the temperature $T_{0}$ of the air; the temperature must be achieved by the body cooling until the cooling phenomenon is completed. Both these coordinates $\left(t_{0}, T_{0}\right)$ determine the position of the final point of the curve $T=f(t)$, existing on the temperature stable potential field $(S P F)_{T}$.

Therefore $T_{0}$ should be substituted instead of the variable $T$, and $t_{0}$ in the place of $t$. Furthermore, after performing some simple algebraic operations, one obtains

$$
t_{0}=\Theta \ln 2
$$

The rate $v$ of the body cooling, as the first (first order) derivative of the temperature $T$ against time $t$, may be determined.

Here is the mathematic configuration:

$$
v=-\frac{d T}{d t}=\frac{2\left(T_{1}-T_{0}\right)}{\Theta} e^{-\frac{t}{\Theta}}=v_{1} e^{-\frac{t}{\Theta}}
$$

The coordinates of the final points of the curve of this magnitude, situated on $(A P F)_{T}$ and $(S P F)_{T}$, are determined by substituting $t=0$, and then $t=t_{0}=\Theta \ln 2$ to (24). After doing that operation, one obtains

$$
\begin{aligned}
& v_{1}=\frac{2\left(T_{1}-T_{0}\right)}{\Theta} \\
& v_{0}=\frac{v_{1}}{2}=\frac{T_{1}-T_{0}}{\Theta}
\end{aligned}
$$

The image of the dependence $v=f(t)$ is the decreasing exponential curve (Fig. 5), approaching the horizontal asymptote, positioned on the time axis of the coordination system $0 t T$.

The real variable course of the body cooling rate takes place for $0 \leq t \leq t_{0}$, and further (for $t>0$ ) that magnitude assumes the zero value.

That means the temperature of the body and air is common, and invariable, constant (see Fig. 4) in this time variability interval.

From the mathematic point of view the derivative of temperature against time is reduced to zero. 


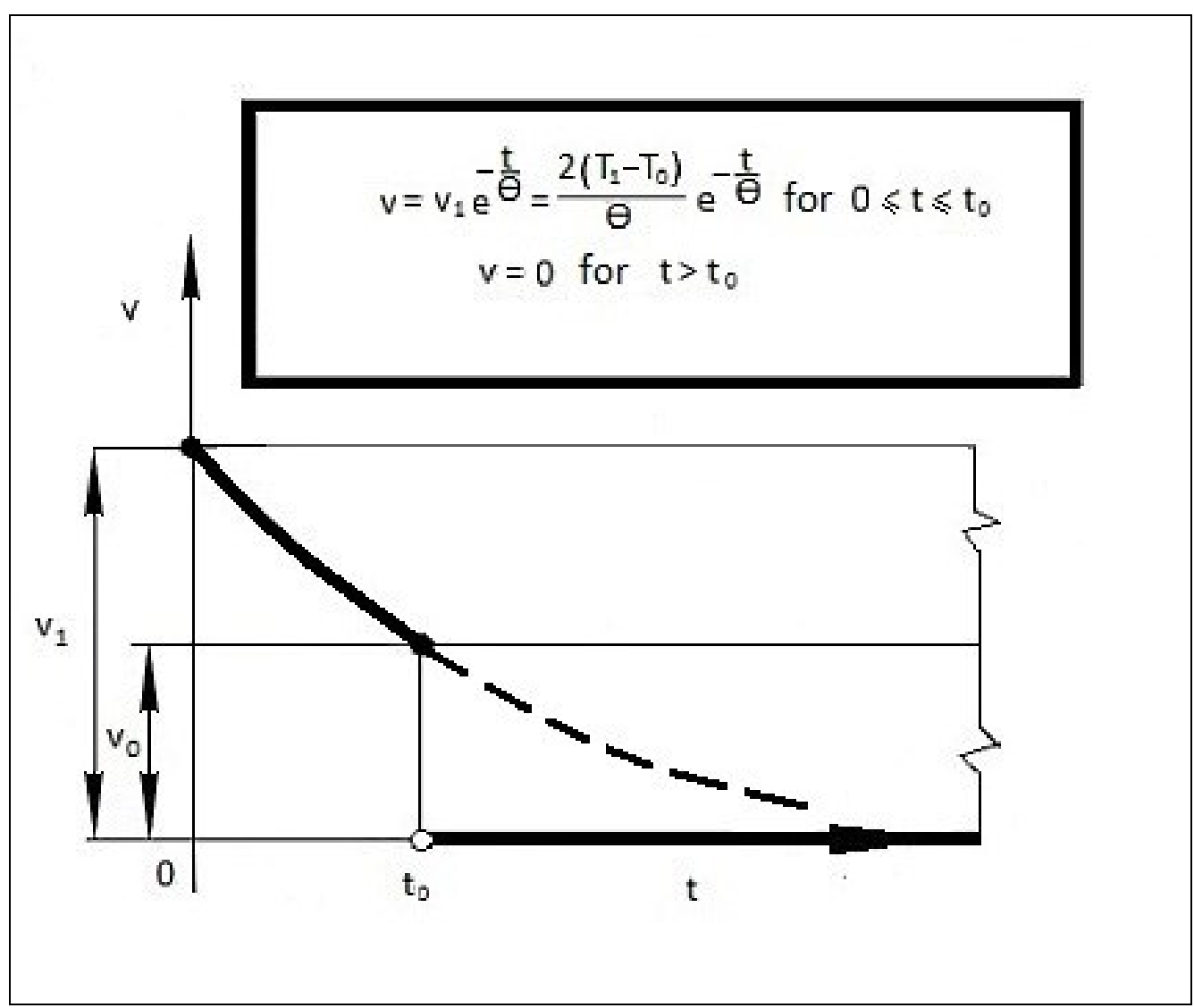

Fig. 5. Graphical image of the adequate dependence of body cooling rate on time of the phenomenon lasting.

For comparative purposes, now it is worth presenting the form of dependence of the cooling rate $v$ on temperature $T$ of the cooled body. Therefore, first the numerator of the formula (24) was determined, by expressing it as the function of $T$, based on the dependence (22), to obtain

$$
2\left(T_{1}-T_{0}\right) e^{-\frac{t}{\Theta}}=T+T_{1}-2 T_{0}
$$

Then, by substituting it to (24), one finally obtains

$$
v=\frac{T_{1}-2 T_{0}}{\Theta}=\frac{1}{\Theta} T
$$

or

$$
v=-\frac{d T}{d t}=v_{0}^{*}+k T
$$


where $v_{0}^{*}$ denotes an apparent, non-real initial velocity (negative as to its value), and $k-$ directional constant (directional coefficient), with the unit of the first magnitude being ${ }^{o} C \cdot s^{-1}$, whereas $s^{-1}$ of the second one.

The graphic illustration of that dependence (Fig. 6) shows that it is a straight line, with the directional coefficient $k=\Theta^{-1}$ being positive. The solid line denotes the real course of the rate, whereas the dashed line determines the apparent (mathematically) positioning of that magnitude. Thus it is physically determined only in the interval $T \in\left\langle T_{0}, T_{1}\right\rangle$.

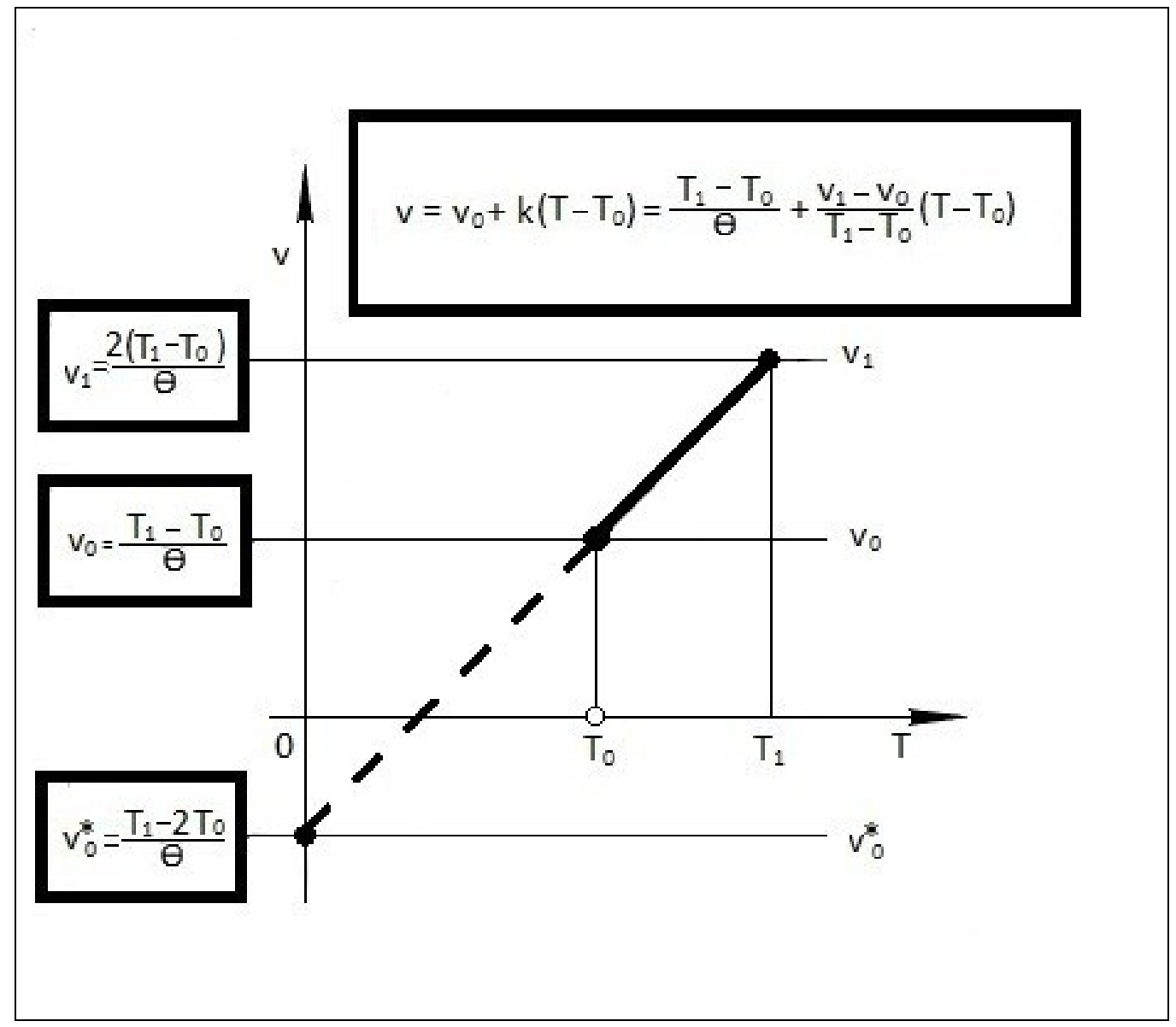

Fig. 6. Indicative adequate dependence of the cooling rate of a body on its temperature.

For the terminal values of this interval, the corresponding rates $v_{0}, v_{1}$ may be determined by substituting these two pairs of magnitudes, i.e. $v_{0}, T_{0}$ and $v_{1}, T_{1}$ to the equation (28). It is obtained through the following:

$$
\begin{aligned}
& v_{0}=\frac{T_{1}-2 T_{0}}{\Theta}+\frac{1}{\Theta} T_{0}=\frac{T_{1}-T_{0}}{\Theta} \\
& v_{1}=\frac{T_{1}-2 T_{0}}{\Theta}+\frac{1}{\Theta} T_{1}=\frac{2\left(T_{1}-T_{0}\right)}{\Theta}
\end{aligned}
$$


Thus the adequate equation of the considered phenomenon should possess the following form:

$$
v=v_{0}+k\left(T-T_{0}\right)
$$

where

$$
k=\frac{v_{1}-v_{0}}{T_{1}-T_{0}}
$$

Taking into account (33) and (30) in the formula (32), one obtains the dependence $v=f(T)$ relating to all parametric characteristics of the phenomenon. Therefore

$$
v=\frac{T_{1}-T_{0}}{\Theta}+\frac{v_{1}-v_{0}}{T_{1}-T_{0}}\left(T-T_{0}\right)
$$

It appears now, there are the essential differences between the existent and those new descriptions of the analysed system. The new descriptions of this thermal phenomenon (body cooling in the air) possess the adequate character, which has not been visible in the existent outcome interpretation version (Newton's law) and its developments.

All these differences result from the fact that the phenomenon of body cooling was described on different cognitive stages. The adequate, presented in this work, description took its beginning at the very source. This is why it has a proper form and the contents. It is worth noting the determination of real variability interval of such magnitudes like the temperature of the cooled body and the cooling rate. It has been also discovered that the final cooling rate is not equal to zero, but possesses a determined value, equal to the half of the initial rate, which possesses the cooled body. Coming to some important conclusion, one should note, the infinitely lasting of the body cooling phenomenon, presented in the existent records, is not possible.

\section{CONCLUSION}

In the conclusion it should be underlined the essential significance of the source differential equation. It is a starting point to the adequate description of the phenomenon of body cooling in the air. The solution of such an equation refers to the real thermal space-time, limited by the real potential fields. The air temperature, determining the position of one of these fields, is not an asymptote, the fact that takes place in the Newton's model, thus suggesting the body may be cooled down (assuming the air temperature) not earlier than after an infinitely long time.

In summing up the work, it is worth comparing all functional characteristics of the phenomenon of solid cooling in the air in the way it is possible to compare these adequate with the Newton's law and the characteristics derived on its basis. That comparison has been tabulated (Table 1), with the columns presenting the characteristics of type $T=f(t)$, $v=f(t)$, and $v=f(T)$ : adequate and non-adequate in character, that is non-corresponding with the described thermal phenomenon. The thick line is used for the area covering the 
Newton's law; it was the inspiration for the consideration of the presented work with the solution leading to obtain the adequate solutions of the subject problem.

Table 1. Comparison of adequate and inadequate descriptions of the phenomenon of solid cooling in the air.

\begin{tabular}{|c|c|c|}
\hline$T=f(t)$ & $\begin{array}{c}T=T_{0}+\left(T_{1}-T_{0}\right)\left(2 e^{-\frac{t}{\theta}}-1\right) \quad \text { for } 0 \leq t \leq t_{0} \\
T=T_{0} \text { for } t \geq t_{0}\end{array}$ & $T=T_{0}+\left(T_{1}-T_{0}\right) e^{-k t}$ \\
\hline$v=f(t)$ & $v=v_{1} e^{-\frac{t}{\theta}}=\frac{2\left(T_{1}-T_{0}\right)}{\theta} e^{-\frac{t}{\theta} \quad \text { for } 0 \leq t \leq t_{0}}$ & $v=v_{1} e^{-k t}=\frac{T_{1}-T_{0}}{\theta} e^{-k t}$ \\
$v=0 \quad$ for $t>t_{0}$ & $v=k\left(T-T_{0}\right)$ \\
\hline$v=f(T)$ & $v=v_{0}+k\left(T_{1}-T_{0}\right)=\frac{T_{1}-T_{0}}{\theta}+\frac{v_{1}-v_{0}}{T_{1}-T_{0}}$ & \\
\hline
\end{tabular}

Amongst all the characteristics, the functional (Table 2) and parametric ones (Table 3) have been separated. The parametric characteristics are: time constant $\Theta$, determined from the formula (23), that is

$$
\Theta=\frac{t_{0}}{\ln 2}
$$

the final cooling rate $v_{0}$, determined by the formula (3), as well as the initial cooling rate $v_{1}$, covered in the formula (31).

Table 2. Functional characteristics of the phenomenon of solid cooling in the air.

\begin{tabular}{|c|c|c|}
\hline \multirow{2}{*}{$T=f(t)$} & $T=T_{0}+\left(T_{1}-T_{0}\right)\left(2 e^{-\frac{t}{\theta}}-1\right)$ & $0 \leq t \leq t_{0}$ \\
\cline { 2 - 3 } & $T=T_{0}$ & $t \geq t_{0}$ \\
\hline \multirow{2}{*}{$v=f(t)$} & $v=v_{1} e^{-\frac{t}{\theta}}=\frac{2\left(T_{1}-T_{0}\right)}{\theta} e^{-\frac{t}{\theta}}$ & $0 \leq t \leq t_{0}$ \\
\cline { 2 - 3 } & $v=0$ & $t>t_{0}$ \\
\hline \multirow{2}{*}{$v=f(T)$} & $v=v_{0}+k\left(T_{1}-T_{0}\right)=\frac{T_{1}-T_{0}}{\theta}+\frac{v_{1}-v_{0}}{T_{1}-T_{0}}$ & $T_{0} \leq T \leq T_{1}$ \\
\cline { 2 - 3 } & $v_{1}=v_{0}$ & $T=T_{0}$ \\
\hline
\end{tabular}


Table 3. Parametric characteristics of the phenomenon of solid cooling in the air.

\begin{tabular}{|c|}
$\theta=\frac{t_{0}}{\ln 2}$ \\
$v_{0}=\frac{T_{1}-T_{0}}{\theta}=\frac{T_{1}-T_{0}}{t_{0}} \ln 2$ \\
$v_{1}=\frac{2\left(T_{1}-T_{0}\right)}{\theta}=\frac{2\left(T_{1}-T_{0}\right)}{\theta} \ln 2$ \\
\hline
\end{tabular}

The contents' analysis of the two former Tables indicates that only three parameters: entrance parameter, being the initial body temperature, that is $T_{1}$; two exit/outcome parameters, being the time, completed cooling time $t_{0}$ and the final body temperature $T_{0}$ are needed to describe the entire phenomenon of body cooling.

\section{References}

[1] Z. Osiński, Przegląd Mechaniczny, 51(16) (1996) 11-15 (in Polish).

[2] W. Bogusz, J. Giergiel, S. Kasprzyk, M. Zabawa, A. Muszyńska, J. Muszyński, B. Radziszewski, J. Szadkowski, Problems of stability in discrete systems. Non-linear vibrations, PWN, Warszawa 1971, 9-52 (in Polish).

[3] Z. Dąbrowski, Przegląd Mechaniczny 62(11) (2007) 15-18 (in Polish).

[4] A. Momber, R. Kovacevic, Principles of Abrasive Water Jet Machining, SpringerVerlag Berlin Heilderberg New York, 1988.

[5] A. Momber A., Kovacevic R., Manufacturing Science and Engineering 68(1) (1994) 361-366.

[6] E. Wantuch, R. Kot, Problem of projection accuracy of curvilinear path under treatment using high-pressure water-abrasive jet system (in Polish). Proc. of 26th Sci. School on Abrasive Treatment, Łódź, September 2003, 329-337.

[7] E. Wantuch, P. Dudek, T. Jarosz, R. Kot, Mechanik 79(4) (2006) 266-271 (in Polish).

[8] P. Słowik, W. Zowczak, Mechanik 81(11) (2008) 948-950 (in Polish).

[9] W. Zowczak, Przeglad Elektrotechniczny 7 (2008) 129-131 (in Polish).

[10] W. Wrona, Mathematics, Part 2 (in Polish), PWN, Warszawa 1967. 
[11] Zdzisław Pluta, Fundamentals of surface smoothing by elastic abrasive wheels (in Polish). Wydawnictwo Politechniki Koszalińskiej, Koszalin 2007.

[12] Zdzisław Pluta, Tadeusz Hryniewicz, Int. J. Adv. Manuf. Technol. 51 (2010) 217-223 DOI:10.1007/s 00170-010-2595-5.

[13] Zdzisław Pluta, Tadeusz Hryniewicz, Int. J. Adv. Manuf. Technol. 62(5) (2012) 529-542, DOI:10.1007/s 00170-011-3813-5. 